\title{
Spinal muscular atrophy: epidemiology and health burden in children - a Polish national healthcare database perspective before introduction of SMA-specific treatment
}

\author{
Anna Kostera-Pruszczyk ${ }^{1}$ (D), Łukasz Napiórkowski², Krystyna Szymańska ${ }^{3}$, Joanna Jędrzejczak ${ }^{4}$, \\ Marcin Roszkowski ${ }^{5}$ Jerzy Słowiński ${ }^{6}$, Anna Frączek ${ }^{1}$, Danuta Ryglewicz ${ }^{7}$, Barbara Więckowska ${ }^{2,8}$ \\ ${ }^{1}$ Department of Neurology, Medical University of Warsaw, Warsaw, Poland; ERN EURO-NMD \\ ${ }^{2}$ Department of Analyses and Strategy, Ministry of Healthcare, Warsaw, Poland \\ ${ }^{3}$ Department of Experimental and Clinical Neuropathology, Mossakowski Medical Research Centre, \\ Polish Academy of Sciences, Warsaw, Poland \\ ${ }^{4}$ Department of Neurology and Epileptology, Centre of Postgraduate Medical Education, Warsaw, Poland \\ ${ }^{5}$ Department of Neurosurgery, The Children's Memorial Health Institute, Warsaw, Poland \\ ${ }^{6}$ Medical University of Silesia, School of Public Health, Katowice, Poland \\ ${ }^{7}$ Military Institute of Aviation Medicine, Warsaw, Poland \\ ${ }^{8}$ Social Insurance Department, Warsaw School of Economics, Warsaw, Poland
}

\begin{abstract}
Introduction: Spinal muscular atrophy (SMA) is one of the most frequent autosomal recessive neuromuscular disorders. It leads to progressive muscle weakness, premature death or permanent ventilation. Significant disability, scoliosis, severe pulmonary infections and other problems require in- and outpatient medical care. Various approaches have been used to evaluate SMA epidemiology, healthcare burden and adherence to standard of care. The recent introduction of pharmacological treatment in a large SMA population will change the course of the disease and the healthcare requirements of patients.

Material and methods: We have used the National Health Fund database to identify children with SMA and the healthcare service they received in the pre-pharmacological treatment era. Pivotal phase II and III medical trials for nusinersen were conducted between 2013 and 2015. The National Treatment Programme of SMA patients with nusinersen in our country was started in January 2019. The year 2014 was used to evaluate incident cases.

Results: 51 new SMA cases (incidence 1:7,356) and 518 SMA patients younger than 18 were identified in 2014.32 (6.2\%) deaths were recorded, half in the first two years of life. $35(6.8 \%)$ patients received palliative and $115(22.2 \%)$ long-term care (including assisted ventilation). A total number of 3,057 days of hospital stay were reported. Only $65 / 518(12.6 \%)$ patients did not receive publicly-funded healthcare service other than specialist or general practitioner's consultation.

Conclusions: SMA caused significant mortality and morbidity in children. The National Health Fund database can be used to reliably record incident cases and track the care provided to paediatric SMA patients.

Key words: spinal muscular atrophy, epidemiology, healthcare burden, incidence

(Neurol Neurochir Pol 2021; 55 (5): 479-484)
\end{abstract}

Address for correspondence: Anna Kostera-Pruszczyk, Department of Neurology, Medical University of Warsaw, Banacha 1a, 02-097 Warsaw, Poland; e-mail: anna.kostera-pruszczyk@wum.edu.pl

Received: 30.03.2021 Accepted: 6.09.2021 Early publication date: 19.10.2021

This article is available in open access under Creative Common Attribution-Non-Commercial-No Derivatives 4.0 International (CC BY-NC-ND 4.0) license, allowing to download articles and share them with others as long as they credit the authors and the publisher, but without permission to change them in any way or use them commercially. 


\section{Introduction}

Spinal muscular atrophy (SMA) is one of the most frequent autosomal recessive neuromuscular disorders. SMA is caused by mutations of the survival motor neuron (SMN1) gene located on chromosome $5 q$ [1]. Depending on the severity of symptoms, three SMA forms have been defined by the International SMA Consortium: severe infantile SMA1 manifested in the first six months of life and diagnosed in children never able to sit unsupported; SMA2 in children who never walk; and SMA3 in those who achieve walking ability. SMA4 is the mildest, and rare, form, and becomes manifest clinically in the second or third decades of life [2]. Overall, almost all patients with SMA are symptomatic and diagnosed in childhood [3]. Access to genetic testing allows precise SMA diagnosis: approximately $97 \%$ of patients in our country have exon 7 biallelic deletion of SMN1 [4]. In the remainder, point mutations in SMN1 have been identified [5].

SMA is a disease with an incidence of approximately 1:3,900-16,000 live births, as evaluated by direct contact with genetic laboratories across Europe [6]. As a diagnosis of SMA requires genetic confirmation, such a study provides accurate epidemiological data. In the era of emerging SMA therapies, it is important to follow the disease course and the access of patients to healthcare services. One way of doing this is via the use of disease-specific registries, such as the global SMA TREAT NMD registry [7]. Another option is the use of healthcare or insurance databases.

There are several major indicators of the disease course in SMA. SMA1 leads to respiratory insufficiency or death during the first two years of life in the majority of patients. SMA2 and SMA3 can also lead to respiratory compromise later in life and cause significant disability. Scoliosis is seen in most SMA2 and SMA3 patients [3]. Until recently, only symptomatic treatment could be offered [8]. Physiotherapy is indicated in all SMA patients. Scoliosis surgery, respiratory support, gastrostomy (PEG) or gastric fundoplasty are indicated in advanced clinical stages $[9,10]$. A recent report on the standard of care received by SMA patients was collected by the TREAT NMD registry [7].

\section{Clinical rationale for study}

The aim of our study was to use the national insurance database to estimate disease incidence, identify juvenile SMA patients, and analyse the medical care they received when no treatment for SMA was available worldwide. Pivotal phase II and III medical trials for nusinersen, the first drug registered for SMA treatment, were conducted between 2013 and 2015 [11-13]. The results of these trials led to the approval of the drug in 2016 by the U.S. Food and Drug Administration (FDA) and in 2017 by the European Medicines Agency (EMA). The Polish National Treatment Programme of SMA patients with nusinersen started in January 2019. Nusinersen is currently reimbursed for all patients, regardless of age and SMA type. The next two drugs for SMA treatment registered in the USA or Europe are risdiplam and onasemnogene abeparvovec-xioi [14]. These are currently not reimbursed in Poland. Additionally, a newborn screening programme for SMA was started in April 2021.

\section{Material and methods}

We analysed the National Health Fund (NFZ) registry. This national insurance database is principally used for financial settlement of health services by the NFZ, and therefore it contains only healthcare services covered by public funding. Complete data was available for the period 1 January 2009 to 31 December 2015 supplemented with dates of deaths until 21 April 2016. The analyses were conducted for patients treated in 2014, and compared to health services in 2013.

Data was collected on SMA patients younger than 18 . ICD10 code G12 was used to identify SMA patients. The code was not restricted to G12.0 (Infantile spinal muscular atrophy, type I [Werdnig-Hoffman]), as the reporting system allows the reporting of a three-character ICD10 code (instead of five-character code) in cases other than inpatient treatment. Only the primary diagnosis code at discharge was taken into account. Age cut-off was used for two reasons: in Poland, all minors are covered by insurance regardless of the employment status of their caregivers, so we assumed that all healthcare services will be recorded. Secondly, all SMA1, SMA2 and most SMA3-4 patients are diagnosed in this age group, allowing us to identify incident cases in 2014.

An SMA patient is defined for the purposes of this study as a person who was recorded at least twice in the NFZ database with an ICD10 G12* code or, for acute cases, only once and who died within two years of their first appearance. The rule was based on the assumptions that SMA patients should regularly visit a physician or attend physiotherapy and that the first outpatient consultation or hospital stay begins the diagnostic process which can confirm the diagnosis of SMA - then more services will be recorded with the same ICD10 code, or, less frequently, an alternative diagnosis can be established (there is only a single public healthcare service with a $\mathrm{G} 12^{*}$ code).

After an SMA patient was identified, we used her or his unique identifier to follow all healthcare services (the reported ICD10 code was not taken into account), including hospitalisations (ward, length of stay, surgical procedures for scoliosis or gastrostomy tube placement), specialised outpatient consultations, palliative or long-term care, physiotherapy, invasive (IV) or noninvasive (NIV) ventilation, or death. All deaths up to 21 April 2016 were considered in order to measure the death rate in SMA patients.

\section{Results}

We identified 51 new SMA cases in 2014. 15 children (29.4\%) were diagnosed in the first year of life and $41.2 \%$ within the first two years. There were 375,200 live births in 


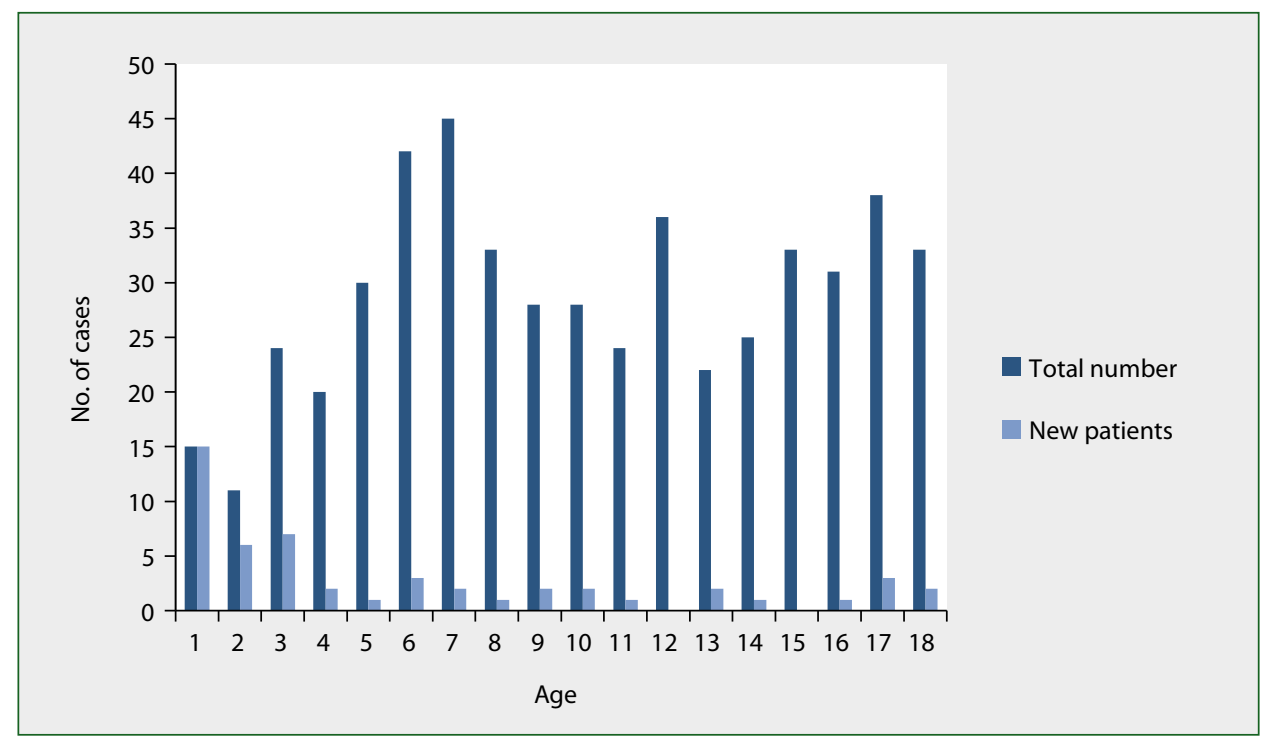

Figure 1. Age distribution at diagnosis date and total number of patients by age in 2014

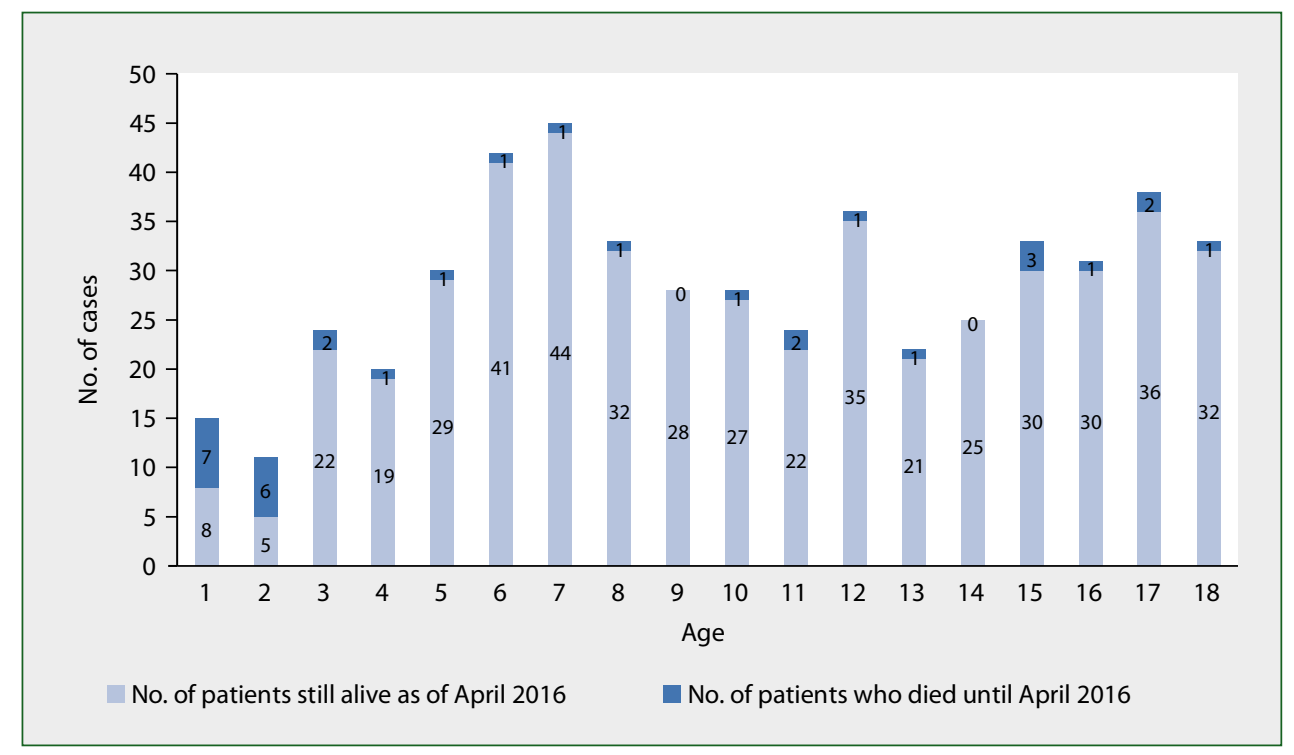

Figure 2. Age distribution and number of deaths per year of age

2014, according to the Central Statistical Office (GUS), yielding an incidence of 1:7,356. The age distribution at diagnosis date and total number of patients by age for 2014 is presented in Figure 1.

There were 518 SMA patients younger than $18 ; 39.8 \%$ were girls. A total of 32 deaths was recorded in 2014 (6.2\%). The death rate was highest in the first two years of life, accounting for $50 \%$ (13/26 cases) of children in this group (Fig. 2). Age distribution and number of deaths per each year of age is presented in Figure 2.

The results of the analysis indicate that in- or outpatient publicly-funded physiotherapy was provided to $273 / 518$ (52.7\%) patients in 2014. Additionally, 35 (6.8\%) patients received palliative care (defined by the NFZ as comprehensive care for patients suffering from incurable, progressive and life-limiting illnesses aimed at improving the quality of life, preventing pain and other somatic symptoms) and $115(22.2 \%)$ received long-term care (this includes assisted ventilation and other services for patients with chronic illnesses or disabilities who need constant non-hospital medical care). Overall, $74.3 \%$ received physiotherapy, palliative care or long term care.

The method of reporting mechanical ventilation used in 2013 did not allow us to separate NIV from IV. Any kind of ventilation was received by 99 (19.7\%) children in 2013. In 2014, ventilation was provided for $112(21.6 \%)$ patients: 


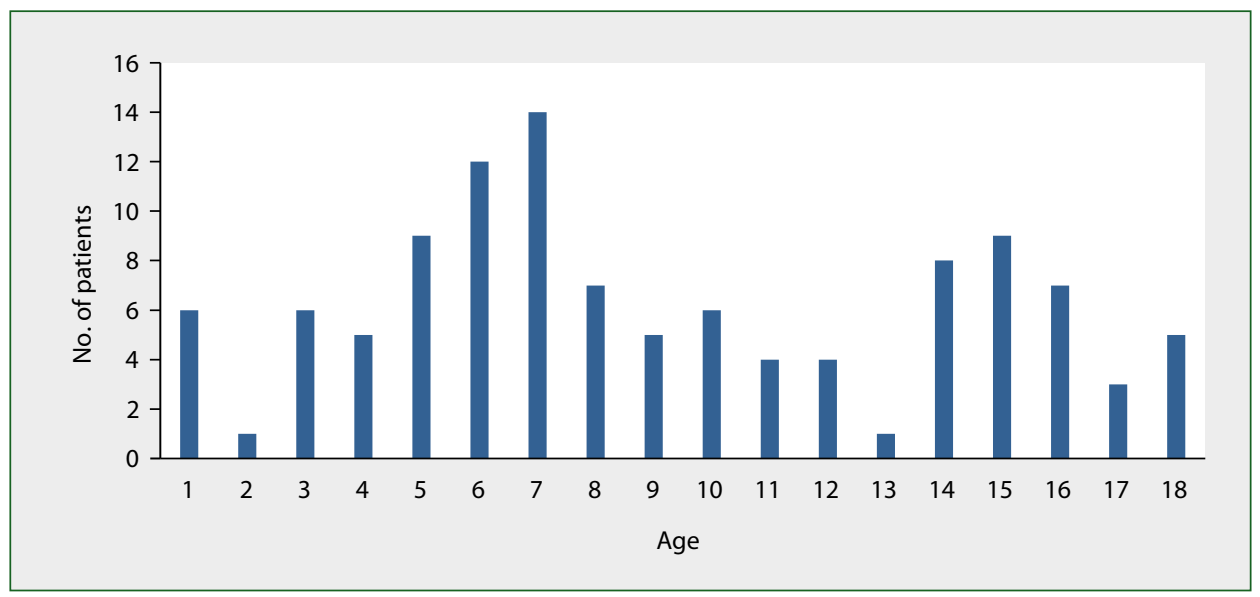

Figure 3. Age distribution of patients receiving mechanical ventilation in 2014

38 (34\% of all ventilated) patients received NIV, while IV was reported in 98 , indicating that 24 patients were transferred from NIV to IV during that year. NIV was provided to 51 (38\%) of 133 receiving ventilator support in 2015 .

The age distribution of the patients receiving mechanical ventilation in 2014 is presented in Figure 3.

\section{Hospitalisations}

There were 321 hospital stays of SMA paediatric patients in 2013 with a total of 3,057 days of stay, while 313 hospitalisations accounted for 2,883 days of stay in 2014. The average length of stay (ALOS) was similar in both years, and amounted to 9.5 days in 2013 and 9.2 days in 2014. Median length of stay (MLOS) remained unchanged at 4 days.

Most hospital stays in 2013 and 2014 were reported by the paediatric ( $43.6 \%$ and $42.5 \%$ respectively), intensive care $(11.8 \%$ and $12.8 \%)$, and paediatric neurology departments (8.4\% and $7.3 \%)$.

We evaluated two surgical procedures that are related to the progressive course of SMA: gastrostomy and scoliosis surgery. Gastrostomy and scoliosis surgery were reported for four and 11 children in 2013, 10 and 10 children in 2014, and 10 and 13 children in 2015 , respectively. Scoliosis surgery was recorded only in children aged six and above.

\section{Specialised outpatient care}

There were 1,648 and 1,638 outpatient visits reported in 2013 , and 2014 , respectively, with $27.3 \%$ and $28.4 \%$ of visits provided by the paediatric neurology or neurology service. Outpatient geneticists' consultations were received by 48 patients in 2013 and 40 in 2014. The number of specialised outpatient care visits does not include the general paediatric service that is usually provided by primary care physicians. For only 65 (12.6\%) patients, no healthcare service other than specialist or general practitioner consultation was recorded.

\section{Discussion}

Healthcare databases can be used as surrogate tools in epidemiological studies, if a precise coding method can be applied to a well-defined population [15]. We undertook such an effort for SMA minors in Poland. All of them are covered by health insurance (NFZ) which records all publicly-funded healthcare services. We decided to analyse data from the period when no SMA-specific treatment options had been available. We identified 51 new SMA cases in 2014, corresponding with an incidence of 13.6 per 100,000, or 1 per 7,356 live births. This is in good agreement with a recent SMA epidemiological study conducted by direct contact with genetic laboratories across Europe. With this methodology, SMA incidence in Poland in 2015 was 13.5 per 100,000 (95\% CI 10.1-17.6) [6]. In our previous study based on information collected from genetic laboratories, the incidence of all SMA subtypes was 1 per 7,127 in Warsaw and 1 per 9,320 across Poland, suggesting underdiagnosis in some regions of the country [16]. Our current results probably indicate improved SMA diagnosis [6, $16,17]$. A recent literature review estimates that SMA1 patients constitute $50-60 \%$ of new cases, with an incidence of 5.5 per 100,000 live births [17]. In the study by Jedrzejowska et al. [16] in 2010, the incidence of SMA1 patients in Poland was 3.2 per 100,000 newborns.

The methodology we currently use does not allow us to verify SMA subtype. 15 patients (only $30 \%$ ) were diagnosed in the first year of life (incidence of 3.99 per 100,000 live births). This might be explained either by diagnostic delay — with some SMA1 children diagnosed after 12 months of life, or a higher proportion of SMA2-3 patients in our cohort. Genetic testing of SMN1 gene deletions and point mutations has been available in our country for many years $[4,18]$. As SMA1-3 patients are diagnosed during childhood, we assume that the NFZ database identified all, or the vast majority, of SMA1-3 patients.

Most of the information on the medical care in the large SMA patient cohort comes from registries such as the global 
TREAT NMD registry [7]. It is estimated that the registry population in the analysed period was at least half that of the true SMA population $[6,19]$. Moreover, the most severe SMA1 patients were possibly underrepresented in the registries, as the disease rapidly progresses and many patients die or become ventilator-dependent in the first years of life $[7,20]$.

In our cohort, 15 (30\%) of the patients were diagnosed in the first year of life and 21 (41\%) within the first two years. We assume that most of the children diagnosed in the first two years of life represent acute SMA, as seven (33\%) of them received ventilatory support and $50 \%$ of all recorded deaths occurred in children younger than two, reflecting the natural course of SMA1 $[21,22]$. In the study by Farrar et al., survival for SMA1 children at 1,2, 4 and 10 years was $40 \%$, $25 \%, 6 \%$ and $0 \%$, respectively [20]. The survival probabilities of SMA2 patients at 1,2, 4, 10, and 20 years are $100 \%$, $100 \%, 100 \%, 92 \%$, and $92 \%$, while SMA3 patients generally have normal life expectancy [23]. Overall mortality before the $18^{\text {th }}$ birthday in our study was $6.2 \%$. Although proactive care and NIV or IV ventilation increase survival in SMA1, mortality in this age group can be attributed to SMA1 or SMA2 [2, 3, 20, 24].

SMA patients require multidisciplinary care. Standard of care (SoC) was first published in 2007, and updated in 2016 [8, 25]. Adherence to the current standard of care is a goal for all SMA patients. The TREAT NMD registry study analysed implementation of the $2007 \mathrm{SoC}$ and demonstrated significant variability in participating countries, especially with regard to the preferred method of ventilatory support (NIV vs. IV) [7]. NIV is advocated for patients with neuromuscular diseases [26]. In SMA1 children, it not only improves survival but facilitates development of speech as well [24]. In our cohort, $30.5 \%$ of children received ventilatory support. The choice of IV vs. NIV depends on many variables, including the approach to end of life decisions by the patient's parents, the organisation of healthcare systems, and also the physician's personal preferences. Although IV was reported in the majority of cases, there was a trend towards more frequent use of NIV between 2014 and 2015.

Our analysis revealed that approximately 10 children per year had scoliosis surgery between 2013 and 2015. This is a similar proportion to scoliosis surgery performed per year in SMA patients reported in a study from the paediatric USA KID Healthcare Cost and Utilisation Project covering administrative data from over 3,500 US hospitals in 36 states [27]. The number of scoliosis surgeries registered by the NFZ database was higher than the total number reported by patients recruited from our country to the TREAT NMD database at the time of previous analysis [7].

We conclude that the National Health Fund database can be used as a tool to monitor incident cases in children with SMA and the healthcare services they receive. The structure of the database will allow us to follow newly identified patients from the health service perspective.
Our data shows that in 2014 not all SMA children had access to care coordinated by a neurologist or paediatric neurologist, and a substantial number of patients did not receive state-funded physiotherapy. The proportion of patients treated with NIV was still much lower than IV.

\section{Clinical and implications and future directions}

Our analysis can serve as a reference for the change in the clinical course and healthcare requirements of patients with this devastating disease brought about by disease-modifying therapy and the implementation of newborn screening for SMA.

\section{Limitations of study}

We evaluated only paediatric SMA patients, as in this age group $100 \%$ of patients are covered by insurance in our country and there is a very low risk that the $\mathrm{G} 12^{*}$ code will be related to other than an SMA diagnosis (e.g. ALS). We had no opportunity to verify diagnostic delay or SMA subtype. Although currently all palliative care, long term care and hospitalisations are publicly-funded, some outpatient healthcare services are available in the private sector as well (specialist consultations or physiotherapy). These are not reported to the NFZ database, and could not be included in the analysis.

Acknowledgments: This study was supported by a project 'Healthcare maps - System Analyses and Implementation Database', implemented by the Department of Analyses and Strategy of the Ministry of Health, co-financed from European Union funds under the European Social Fund. Its purpose is to provide evidence-informed healthcare policy in Poland [28]. Conflict of interest: None.

\section{References}

1. Lefebvre S, Bürglen L, Reboullet S, et al. Identification and characterization of a spinal muscular atrophy-determining gene. Cell. 1995; 80(1): 155-165, doi: 10.1016/0092-8674(95)90460-3, indexed in Pubmed: 7813012.

2. Zerres K, Davies KE. 59th ENMC International Workshop: Spinal Muscular Atrophies: recent progress and revised diagnostic criteria 1719 April 1998, Soestduinen, the Netherlands. Neuromuscul Disord. 1999; 9(4): 272-278, doi: 10.1016/s0960-8966(99)00016-4, indexed in Pubmed: 10399757.

3. Zerres K, Rudnik-Schöneborn S, Forrest E, et al. A collaborative study on the natural history of childhood and juvenile onset proximal spinal muscular atrophy (type II and III SMA): 569 patients. J Neurol Sci. 1997; 146(1): 67-72, doi: 10.1016/s0022-510x(96)00284-5.

4. Jedrzejowska M, Wiszniewski W, Zimowski J, et al. Application of a rapid non-invasive technique in the molecular diagnosis of spinal muscular atrophy (SMA). Neurol Neurochir Pol. 2005; 39(2): 89-94, indexed in Pubmed: 15871052.

5. Wirth B, Herz M, Wetter A, et al. Quantitative analysis of survival motor neuron copies: identification of subtle SMN1 mutations in patients with spinal muscular atrophy, genotype-phenotype correlation, and 
implications for genetic counseling. Am J Hum Genet. 1999; 64(5): 1340-1356, doi: 10.1086/302369, indexed in Pubmed: 10205265.

6. Verhaart IEC, Robertson A, Leary R, et al. A multi-source approach to determine SMA incidence and research ready population. J Neurol. 2017; 264(7): 1465-1473, doi: 10.1007/s00415-017-8549-1, indexed in Pubmed: 28634652.

7. Bladen $\mathrm{CL}$, Thompson R, Jackson JM, et al. Mapping the differences in care for 5,000 spinal muscular atrophy patients, a survey of 24 national registries in North America, Australasia and Europe. J Neurol. 2014; 261(1): 152-163, doi: 10.1007/s00415-013-7154-1, indexed in Pubmed: 24162038.

8. Wang CH, Finkel RS, Bertini ES, et al. Participants of the International Conference on SMA Standard of Care. Consensus statement for standard of care in spinal muscular atrophy. J Child Neurol. 2007; 22(8): 1027-1049, doi: 10.1177/0883073807305788, indexed in Pubmed: 17761659.

9. Finkel RS, Mercuri E, Meyer OH, et al. SMA Care group. Diagnosis and management of spinal muscular atrophy: Part 2: Pulmonary and acute care; medications, supplements and immunizations; other organ systems; and ethics. Neuromuscul Disord. 2018; 28(3): 197-207, doi: 10.1016/j.nmd.2017.11.004, indexed in Pubmed: 29305137.

10. Mercuri E, Finkel RS, Muntoni F, et al. SMA Care Group. Diagnosis and management of spinal muscular atrophy: Part 1: Recommendations for diagnosis, rehabilitation, orthopedic and nutritional care. Neuromuscul Disord. 2018; 28(2): 103-115, doi: 10.1016/j. nmd.2017.11.005, indexed in Pubmed: 29290580.

11. Finkel RS, Chiriboga CA, Vajsar J, et al. Treatment of infantile-onset spinal muscular atrophy with nusinersen: a phase 2, open-label, dose-escalation study. Lancet. 2016; 388(10063): 3017-3026, doi: 10.1016/S0140-6736(16)31408-8, indexed in Pubmed: 27939059.

12. Darras $B T$, Farrar MA, Mercuri $E$, et al. An integrated safety analysis of infants and children with symptomatic spinal muscular atrophy (SMA) treated with nusinersen in seven clinical trials. CNS Drugs. 2019; 33(9): 919-932, doi: 10.1007/s40263-019-00656-w, indexed in Pubmed: 31420846.

13. Mercuri E, Darras BT, Chiriboga CA, et al. CHERISH Study Group. Nusinersen versus sham control in later-onset spinal muscular atrophy. N Engl J Med. 2018; 378(7): 625-635, doi: 10.1056/NEJMoa1710504, indexed in Pubmed: 29443664.

14. Jędrzejowska M, Kostera-Pruszczyk A. Spinal muscular atrophy - new therapies, new challenges. Neurol Neurochir Pol. 2020; 54(1): 8-13, doi: 10.5603/PJNNS.a2019.0068, indexed in Pubmed: 31922583.

15. Andersen JB, Engeland A, Owe JF, et al. Myasthenia gravis requiring pyridostigmine treatment in a national population cohort. Eur J Neurol. 2010; 17(12): 1445-1450, doi: 10.1111/j.1468-1331.2010.03089.x, indexed in Pubmed: 20491896.

16. Jedrzejowska M, Milewski M, Zimowski J, et al. Incidence of spinal muscular atrophy in Poland - more frequent than predicted? Neuroepidemiology. 2010; 34(3): 152-157, doi: 10.1159/000275492, indexed in Pubmed: 20090376.
17. Verhaart IEC, Robertson A, Wilson IJ, et al. Prevalence, incidence and carrier frequency of $5 q$-linked spinal muscular atrophy - a literature review. Orphanet J Rare Dis. 2017; 12(1): 124, doi: 10.1186/s13023017-0671-8, indexed in Pubmed: 28676062.

18. Jędrzejowska M, Gos M, Zimowski JG, et al. Novel point mutations in survival motor neuron 1 gene expand the spectrum of phenotypes observed in spinal muscular atrophy patients. Neuromuscul Disord. 2014; 24(7): 617-623, doi: 10.1016/j.nmd.2014.04.003, indexed in Pubmed: 24844453.

19. Norwood FLM, Harling C, Chinnery PF, et al. Prevalence of genetic muscle disease in Northern England: in-depth analysis of a muscle clinic population. Brain. 2009; 132(Pt 11): 3175-3186, doi: 10.1093/ brain/awp236, indexed in Pubmed: 19767415.

20. Farrar MA, Vucic S, Johnston HM, et al. Pathophysiological insights derived by natural history and motor function of spinal muscular atrophy. J Pediatr. 2013; 162(1): 155-159, doi: 10.1016/j. jpeds.2012.05.067, indexed in Pubmed: 22809660.

21. Finkel RS, McDermott MP, Kaufmann $P$, et al. Observational study of spinal muscular atrophy type I and implications for clinical trials. Neurology. 2014; 83(9): 810-817, doi: 10.1212/ WNL.0000000000000741, indexed in Pubmed: 25080519.

22. Cobben JM, Lemmink HH, Snoeck I, et al. Survival in SMA type I: a prospective analysis of 34 consecutive cases. Neuromuscul Disord. 2008; 18(7): 541-544, doi: 10.1016/j.nmd.2008.05.008, indexed in Pubmed: 18579378.

23. Chung BHY, Wong VCN, Ip P. Spinal muscular atrophy: survival pattern and functional status. Pediatrics. 2004; 114(5): e548-e553, doi: 10.1542/peds.2004-0668, indexed in Pubmed: 15492357.

24. Bach JR, Saltstein K, Sinquee D, et al. Long-term survival in Werdnig-Hoffmann disease. Am J Phys Med Rehabil. 2007; 86(5): 339348, doi: 10.1097/PHM.0b013e31804a8505, indexed in Pubmed: 17449977.

25. Finkel RS, Sejersen T, Mercuri E, et al. ENMC SMA Workshop Study Group. 218th ENMC International Workshop: revisiting the consensus on standards of care in SMA Naarden, The Netherlands, 19-21 February 2016. Neuromuscul Disord. 2017; 27(6): 596-605, doi: 10.1016/j.nmd.2017.02.014, indexed in Pubmed: 28392274.

26. Bach JR, Gonçalves MR, Hon A, et al. Changing trends in the management of end-stage neuromuscular respiratory muscle failure: recommendations of an international consensus. Am J Phys Med Rehabil. 2013; 92(3): 267-277, doi: 10.1097/PHM.0b013e31826edcf1, indexed in Pubmed: 23051760.

27. Barsdorf Al, Sproule DM, Kaufmann P. Scoliosis surgery in children with neuromuscular disease: findings from the US National Inpatient Sample, 1997 to 2003. Arch Neurol. 2010; 67(2): 231-235, doi: 10.1001/archneurol.2009.296, indexed in Pubmed: 20142532.

28. Więckowska B. Healthcare Needs Maps - Evidence Informed Healthcare Policy. Journal of Health Policy and Outcomes Research. 2017; 1(1): 14-20, doi: 10.7365/JHPOR.2017.1.2. 